\title{
The Application of Piano Accompaniment in Folk Music
}

\author{
Qian Zheng1, a \\ ${ }^{1}$ College of Music, Hengyang Normal University, Hengyang, 421002, China \\ amusic34@126.com
}

\begin{abstract}
Piano accompaniment has a history of more than one hundred years. Chinese national instrumental music has a long history and rich culture, which is an indispensable part in the history of Chinese folk music. With the development and change of modern music, Chinese folk music has entered an era of diversified development. As the "king of Musical Instruments", the combination of piano and Chinese national instrumental music was born by good luck. This paper mainly serious piano accompaniment used in folk music in the production, the status quo, so that more people understand, accept and pay attention to this new things, and even more to develop and innovation, to create more excellent works.
\end{abstract}

Keywords: Piano accompaniment, Folk music, Use of the status quo.

\section{Introduction}

As one of the three major Musical Instruments in the world, the piano, known as the "King of Musical Instruments", has a broad vocal range, rich expressive force, varied harmonic texture and unique timbre beauty. It has been used for hundreds of years as a solo instrument, and is widely used as an accompaniment instrument in orchestral and vocal music. Folk music is the Chinese national instrumental music, is a unique art form in China, it has a long history, rich artistic culture and unique charm, is the treasure of the Chinese nation. With the development and change of modern music and the influx of foreign cultures, Chinese folk music has entered an era of diversified development in order to keep pace with The Times, and the combination of piano accompaniment and folk music has flourished. However, the combination of the two has not been created for a long time. The application of piano accompaniment in national instrumental music has only been practiced for several decades, and it is still a very young discipline. Therefore, most people do not know the value of the combination of the two and how the piano accompaniment is used in folk music. Therefore, through the study on the operation of the piano in the folk music, highlight the value of a combination with the masses, mining, inheritance, development and innovation of folk music and piano music creation enthusiasm, promote the application of piano accompaniment in the folk music, the Chinese national Musical Instruments better inheritance and development, is our obligatory.

\section{The Emergence of Piano Accompaniment in the Use of Folk Music}

\subsection{Background of Generation}

Piano is also often used as an accompaniment instrument to accompany western vocal music and orchestral works in people's sight. The world famous pianist Anton Rubinstein once said: "The piano is a musical instrument, but also a hundred Musical Instruments. This shows the high status and powerful function of the piano.

Chinese national instrumental music with a long history and rich cultural heritage is not accompanied by piano. In the form of accompaniment, different national instrumental music is used to accompany each other or a national band to accompany the solo instrument. However, since the late 1950s and early 1960s, with the development and changes of The Times, the popularity of piano in China, the further communication between Chinese and western music, and the mutual integration of different ethnic music have become an irresistible trend, thus giving birth to the combination of piano accompaniment and Chinese ethnic instrumental music. By our country famous composer -- wen-jin liu's erhu solo, piano accompaniment "ballade of the north", "' feast of sanmenxia lake" is one of the earliest piano and national instrumental music works, because this is the first time by the famous composer in using the western composing techniques in his writing, at the same time making conception creation presided part and the accompaniment, on the basis of retain strong national style, the coordination between the piano and erhu timbre, harmony, differences in style, perfectly with piano and erhu, make both works is not only the piano and the combination of Chinese national instrumental music between model, And has been described by critics as a landmark work. Since then, more and more composers have begun to try to create, so more and more national Musical Instruments began to use piano to accompany the regular score, more and more piano for Chinese national instrumental music works appeared in people's sight, and deeply loved by the masses. They also include He Zhanhao's The Resentment of Lin 'an, Wang Jianmin's Night Mooring at Maple Bridge and The First Erhu Rhapsody.

\subsection{The Characteristics of Piano Accompaniment in Folk Music}

(1) In terms of creation techniques, taking the first two piano accompaniments as examples, they mainly use polyphonic techniques, mainly contrast and imitation polyphonic, and are good at imitating the timbre of different ethnic instrumental music. In the fourth paragraph of these two pieces, the whole chapter greatly uses the gorgeous and delicate contrastive polyphonic technique, which makes the melody lyrical fluently and sets off the solo part beautifully. The bass adopts Canon style imitation technique and pays attention to counterpoint and melody. The same example is the accompaniment of the flute song "Boat Song of the Water 
Township". The long vibrato is used to imitate the free and clear effect of the bamboo flute with the order of strength and weak, which forms a supplementary reply with the long melody of the solo part.

(2) In terms of harmonic texture, since most of China's national instrumental music repertoire adopts the traditional pentatonic mode, the piano also basically uses pentatonic mode to complete the accompaniment. For example, Dong Jinhan used pentatonic arpeggios in the piano accompaniment for the famous Erhu song "Erquan reflecting the moon", vividly displaying the background of "the second spring under the sky" in front of the public, and making the whole music work more fluid. In addition, when the piano accompaniment is used in the folk music, it is not suitable to use multifunctional harmonies, while the discordant second intervals or the fourth and fifth superimposed chords are used throughout the paragraph. For example, when the melody of the erhu jumps and is cheerful, the piano adopts the light and active accompaniment pattern, and frequently uses the collision of large and small second intervals to imitate the joyful temperament of the percussion music, so as to express the vigorous spirit. In the soft and dim lyric part of Erhu, in order to weaken the inner part of harmony to express the artistic conception of the work, complete concordant intervals are used, and parallel fourth and fifth intervals, which are prohibited in harmony rules, are used to create an ethereal feeling. In the texture part of the melody on the left hand low voice part is also a major feature of the piano accompaniment for folk music, often unexpected effect.

(3) Technically, there are a wide variety of Chinese national Musical Instruments. Chinese national Musical Instruments, such as zither, zhongruan and bamboo flute, are mainly composed of horizontal single melodic lines, and most of them are monophonic instruments. Due to the limited number of their own pronounciations, people will feel weak no matter how beautiful the melody is. In contrast, the piano has a prominent longitudinal harmonic texture with a broad range, and is played by ten fingers at the same time. It can produce more than ten notes at the same time, and the timbre is full of changes. Therefore, the piano accompaniment used in national instrumental music can make the sound of musical works more full, the sound effect is more gorgeous and beautiful, and the musical expression is more rich. Secondly, it makes full use of the octave, arpeggios and tremors of the piano in the performance, and makes reasonable use of the pedals to render the mood and foil the atmosphere for the national musical instrument as the main part, so as to achieve a better auditory effect. In this song (see Example 3), the introduction part of the piano plays the 32 minute tremor of 17 bars in the bass area quickly, which highlights a spectacular scene of surging waves and rolling rivers.

\section{The Development of Piano Accompaniment in Folk Music}

\subsection{The Status Quo of Piano Accompaniment in Folk Music}

3.1.1. Application Status of Folk Music Performance

In addition to the classification of eight tones, Chinese national Musical Instruments can be divided into wind instruments plucking instruments, stringed instruments according to their different playing styles. In folk music, the solo instrument is a variety of form of accompaniment, the piano is one of them, and with the piano accompaniment in the application of folk music, more and more people are more likely to choose in the form of the piano performance, since 2007, hosted by China central television (CCTV) national instrumental music television, China's traditional ethnic Musical Instruments, Chinese zither, pipa, erhu, sheng, suona players in solo, most take the form of performance is the piano accompaniment to foil atmosphere, to better show the characteristics of ethnic Musical Instruments. Piano master Lang Lang also worked in the 2007 special Olympics in Shanghai meeting with piano and his father with the original cooperation a classic "horse race", the song written by Lang Lang and deduce the piano accompaniment in person, not only retains the original ethnic and gives the whole song new life, succeed once again be the piano and charm of combination in a national instrumental music show in front of an audience.

Another manifestation of the present situation of piano accompaniment in folk music performance is that one is the combination of Chinese traditional national instrumental music and piano and other western instruments with popular orgizing techniques. The wave of "new folk music" is also springing up and developing rapidly. However, some people believe that the piano, as a western instrument, adopts the temperament system of twelve equal temperament, while the Chinese national instrumental music adopts the five-degree reciprocal temperament to set the tone, and the intonation is a big problem under the two different temperament systems. In this way, the extensive use of piano to accompany folk music in the performance not only leads to the lack of form and connotation.

In short, the use of piano accompaniment in folk music performance is deeply loved by performers and audiences, and the development trend is good. However, there are still some scholars criticizing the use of piano accompaniment in folk music, thinking that piano accompaniment in it seems to be a bit of a preoccupation, lost the original national flavor.

\subsubsection{Application Status of Folk Music Teaching}

Piano accompaniment has not received much attention in the teaching of folk music, but many scholars believe that piano accompaniment should be introduced into the teaching of folk music.

At present, the teaching mode of instrumental music major in colleges and universities is "one-to-one" mode, that is, the cooperation between a student and the piano accompaniment teacher who should be a major is only temporary cooperation when he or she independently seeks for the teacher or classmate of the piano major for the examination. Chinese music is implicit, restrained and pay attention to artistic conception, so most of the music has a large number of introduction, loose board, epilogue and other sections that need to be handled freely, which depends on the player's super high performance level and good musical sensibility to control, but these requirements are undoubtedly very difficult for students. Most students are unable to master the speed and rhythm of the music, and teachers can only train students with the help of metronome, or humming the melody with their mouth, or clapping the rhythm with their hands. However, even in this way, the student's performance still seems mechanical and mechanical. No matter how hard the teacher tries to analyze and demonstrate the works, students still cannot better understand the artistic conception, charm, style and emotion of the works. Therefore, many scholars put forward that piano accompaniment should be added to the class of national instrumental music to guide and pave the 
way for students to learn national instrumental music in class, and help students adjust the rhythm, speed, intonation and other issues. At the same time, it can drive the mood, set off the atmosphere, experience the artistic mood of the work, and better complete the work.

\section{Conclusion}

The national instrumental music is the treasure of Chinese art. With the development of modern music, the national instrumental music also keeps pace with The Times and starts a new development. Piano accompaniment, as a professional music performing art, has rich techniques of expression. With the development and change of modern music and the efforts of composers, piano accompaniment began to be used in folk music. Although we have found some drawbacks through the analysis of the development status quo, national instrumental music has indeed promoted its own development through the combination with piano. Piano accompaniment plays a very active role in folk music and its importance cannot be underestimated. In a word, the combination of piano accompaniment and folk music has complementary advantages in artistic expression, and the perfect combination of the two is a qualitative leap of China's national instrumental music, as well as the result of the integration of Chinese and western musical cultures.

\section{References}

[1] Fan Kunming. Discussion on the Role of Piano Accompaniment in Folk Music [J]. Popular Art, 2013 (14).

[2] Li Xinchun. Thoughts on Piano Accompaniment for Chinese National Instrumental Music [J]. Contemporary Music, 2015 (09).

[3] Liu Peng. The Significance of Piano Accompaniment to Chinese Musical Instrument Performance [J]. Contemporary Music, 2015 (24).

[4] Ning Haitian. Practice and Exploration of Piano Accompaniment Introducing Ruan Le into Teaching [J]. Music World, 2016 (04).

[5] Lu Yang. The Important Role of Piano Accompaniment in National Instrumental Music Performance [J]. Music Time and Space, 2016 (01)

[6] Lv Jingyun. New Theories on Art Psychology [M]. Culture and Art Press, 2000.

[7] Zhang Qian, Wang DiZhao. Fundamentals of Music Aesthetics [M]. Sichuan People's Publishing House, 1983.

[8] SiTu BiChun, Chen Langqiu. Piano Teaching method [M]. Southwest Normal University Press, 2004. 